\title{
Wireless Communication in Dynamic Interference
}

\author{
Malcolm Egan ${ }^{1}$, Laurent Clavier ${ }^{2,3}$, Mauro de Freitas ${ }^{2}$, Louis Dorville ${ }^{4}$, Jean-Marie Gorce ${ }^{1}$ and Anne Savard ${ }^{2,3}$ \\ ${ }^{1}$ Univ. Lyon, INSA Lyon, INRIA, CITI, France \\ ${ }^{2}$ Univ. Lille, CNRS, ISEN, Univ. Valenciennes, UMR 8520, IEMN, France \\ ${ }^{3}$ IMT Lille Douai, Institut Mines Telecom, France \\ ${ }^{4}$ LAMIA Université des Antilles, Guadeloupe (FWI)
}

\begin{abstract}
Fast varying active transmitter sets are a key feature of wireless communication networks with very short transmissions arising in machine-to-machine communications. A consequence is that the interference is dynamic, leading to nonGaussian statistics. In this paper, we study the behavior of largescale communication networks in the presence of isotropic $\alpha$ stable interference, which forms a model for dynamic interference. We first characterize the achievable rate of each link by considering a non-Gaussian input distribution, which is shown to outperform a Gaussian input. Moreover, we analyze the area spectral efficiency, which is the total rate per square meter. Our analysis suggests that analogously to the common model of slowly varying active transmitter sets, dense networks maximize the area spectral efficiency.
\end{abstract}

\section{INTRODUCTION}

Modern wireless communication networks are increasingly heterogeneous. This heterogeneity typically arises from nonuniform placement of base stations and variations in transmit power constraints, which is characteristic of networks employing small-cells and ad hoc networks. Another form of heterogeneity is due to differences in the services that the networks provide. For instance, there are key differences in quantity and type of data, as well as transmission protocols between networks supporting standard cellular or WLAN communication and machine-to-machine (M2M) communications [1].

In standard cellular services, data transmissions typically vary between $1 \mathrm{~KB}$ and $2 \mathrm{MB}$ per transmission for text and image transfers and up to $3 \mathrm{~GB}$ for video transfer [2]. On the other hand, in $\mathrm{M} 2 \mathrm{M}$ communications, data transmission is of the order of $1 \mathrm{MB}$ per month [3]. Transmissions in M2M networks are therefore very short. As a consequence, the active set of transmitting devices at each time can change rapidly.

For a fixed active set of transmitting devices, the interference is well-modeled as Gaussian. This is the basis of the standard interference modeling methodology in wireless cellular networking. An important question therefore arises for M2M networks with a rapidly changing active transmitter set: can the interference be well-modeled as Gaussian? In fact, if the interference is non-Gaussian it will impact performance guarantees and resource allocation, which is often based on the spectral efficiency of each link.

In this paper, we assess the impact of rapidly changing active transmitter sets-or dynamic interference-in largescale M2M networks. We consider a worst-case scenario

This work is supported in part by the ANR ARBurst project. where the network is interference-limited, uncoordinated, and the locations of interferers are governed by a homogeneous Poisson point process. This setup is relevant for networks supporting the internet of things and in large-scale sensor networks, where transmitting devices are very simple and have limited ability to coordinate. We also assume that the active set of transmitters varies symbol-by-symbol, which contrasts with the Gaussian model where the active transmitter set is fixed.

Dynamic interference in wireless networks was introduced in $[4,5]$ by considering a fast-varying (symbol-by-symbol) active transmitter set, with locations governed by a homogeneous Poisson point process. In particular, it was shown that the interfering signal in each time slot is $\alpha$-stable. The $\alpha$-stable interference model is also known to be a good approximation of the true interference distribution when the radius of the network is large, there are no guard zones and the active interferer set changes rapidly [6-8].

The class of $\alpha$-stable random variables are well-known to model impulsive signals, having-unlike Gaussian noiseinfinite variance. Although an expression without a closedform for the error probability in the presence of $\alpha$-stable interference was derived in [4], little is known about the achievable rates and the optimal density of devices in this scenario.

To optimize networks with dynamic interference, we establish a refined interference model which accounts for the statistics of the baseband signal of each interfering device and small-scale fading. As in [4], this leads to interference with isotropic $\alpha$-stable statistics. Applying our recent characterization of the achievable rates of additive isotropic $\alpha$-stable noise channels in [9], we derive a closed-form expression for the achievable rate of the typical user. A key observation is that Gaussian inputs are no longer optimal and that truncated $\alpha$-stable inputs in fact yield higher achievable rates.

A characterization of the achievable rate of the typical user provides a means of establishing the area spectral efficiency [10] of the network, which is the achievable rate per square meter. In particular, we establish an accurate approximation of the area spectral efficiency using truncated $\alpha$-stable inputs. Using this approximation, we find that it is highly desirable to deploy dense networks - consistent with a common strategy in networks based on Gaussian noise models [11]. This suggests that although the optimal signaling differs, the basic network 
architecture of fixed and dynamic interference scenarios is the same.

\section{System MODEL AND INTERFERENCE CHARACTERIZATION}

Consider a large-scale wireless communication network consisting of devices and access points, where each device transmits data to a unique access point. The locations of the devices are governed by a homogeneous Poisson point process (PPP), denoted by $\Phi$, with intensity $\lambda$. We assume that the network is uncoordinated, which forms a worst case model for large-scale M2M communication networks.

For an access point at the origin, $A_{0}$, served by device 0 , the interference at time $t$ from the other devices is given by

$$
I_{t}=\sum_{k \in \Phi \backslash\{0\}} r_{k, t}^{-\eta / 2} h_{k, t} x_{k, t},
$$

where $\eta$ is the path loss exponent of the interfering links. The circularly symmetric complex normal distributed random variable $h_{k, t} \sim \mathcal{C N}(0,1)$ is the Rayleigh fading coefficient for the link from device $k$ to the access point $A_{0}$. The baseband emission of each interferer $k$ is denoted by $x_{k, t}$. We assume that the real and imaginary parts of $h_{k, t} x_{k, t}$ are symmetric and $e^{j \phi} h_{k, t} x_{k, t} \stackrel{(d)}{=} h_{k, t} x_{k, t}$ for all $\phi \in[0,2 \pi)$, which means that $h_{k, t} x_{k, t}$ is isotropic. This is not a strong assumption and is satisfied, for instance, in the case of Rayleigh fading with circularly symmetric complex Gaussian baseband emissions.

The distance of the access point $A_{0}$ from the device at the origin is denoted by $r_{d}$, which is assumed to have a distribution $F_{r_{d}}$, and the location of $A_{0}$ is assumed to be uniformly distributed on the circle of radius $r_{d}$. The signal received by the access point $A_{0}$ at time $t$ is then given by

$$
y_{t}=r_{d, t}^{-\eta / 2} h_{d, t} x_{d, t}+I_{t}+N_{t},
$$

where $h_{d, t} \sim \mathcal{C N}(0,1)$ is the Rayleigh fading coefficient and $x_{d, t}$ is the baseband emission for the typical user. The additive white zero-mean Gaussian noise $N_{t} \sim \mathcal{C N}\left(0, \sigma^{2}\right)$ corresponds to thermal noise at the access point.

In this paper, we are concerned with the scenario where the active interferer set changes rapidly, called dynamic interference. In the worst case scenario, the dynamic interference is (typically non-Gaussian) noise that affects every symbol, which we assume to be independent at each time $t$. There are two key physical mechanisms that can induce dynamic interference. The first mechanism is any protocol where data is transmitted in non-contiguous blocks, which means that interferers do not transmit data continuously.

The second mechanism arises when there are multiple coexisting communication systems, such as IEEE 802.11 (WiFi) and IEEE 802.15 (Zigbee, Bluetooth). The IEEE 802.11 frame is composed of a fixed header of 34 bytes and for a short payload of 250 bytes and data rate of $54 \mathrm{Mbps}$ the on-air time is 42.07 microseconds. On the other hand, the IEEE 802.15 Zigbee frame is 40 bytes with data rate $250 \mathrm{kbps}$, leading to an on-air time of $1.25 \mathrm{~ms}$. Moreover,
Bluetooth is frequency hopping and is present in a 802.15 band only rarely and for a very short time. The result is that Bluetooth and Wi-Fi interferers are active for short periods of time relative to Zigbee transmissions, resulting in dynamic interference. To illustrate the second mechanism Fig. 1 shows the result of an experiment (detailed in [12, Section 2.5.2]) with coexisting Wi-Fi, Bluetooth and Zigbee transmissions. Observe that Bluetooth interference for very short periods of time is sufficient to corrupt a Zigbee transmission.

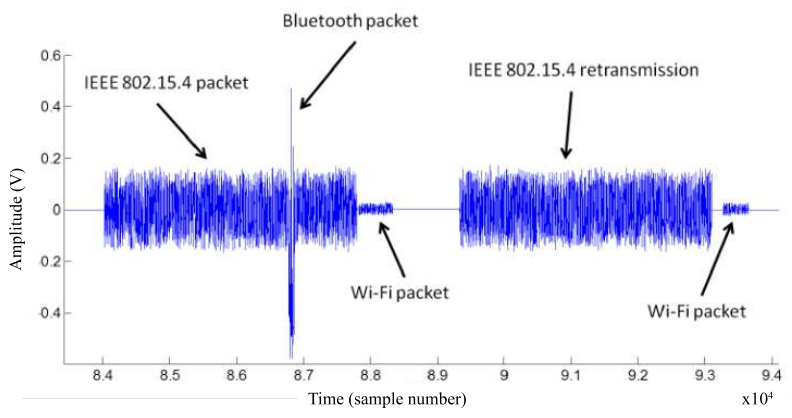

Fig. 1. Coexistence of technologies in the 2.4-GHz band. Measurements made by a National Instruments USRP (detailed in [12, Section 2.5.2]).

Based on these considerations, the received signal by the access point $A_{0}$ in the presence of dynamic interference for the interference-limited setting is the output of a memoryless additive noise channel. In particular, the output $y$ is given by

$$
y=r_{d}^{-\eta / 2} h_{d} x_{d}+I .
$$

Note that we have dropped the time subscript due to the fact that this channel is memoryless. We also remark that device heterogeneity can be captured under the assumption that the probability a device with a given protocol is the same for each device.

In order to evaluate communication in dynamic interference, it is necessary to characterize the statistics of $I$. The basis of this characterization is the theory of isotropic $\alpha$-stable random variables, which we now review.

\section{A. Isotropic $\alpha$-Stable Random Variables}

The $\alpha$-stable random variables are an important class of random variables with heavy-tailed probability density functions, which have been widely used to model impulsive signals [13]. The probability density function of an $\alpha$-stable random variable is parameterized by four parameters: the exponent $0<\alpha \leq 2$; the scale parameter $\gamma \in \mathbb{R}_{+}$; the skew parameter $\beta \in[-1,1]$; and the shift parameter $\delta \in \mathbb{R}$. As such, a common notation for an $\alpha$-stable distributed random variable is $X \sim S_{\alpha}(\gamma, \beta, \delta)$. In the case $\beta=\delta=0$, the random variable $X$ is said to be a symmetric $\alpha$-stable random variable.

In general, $\alpha$-stable random variables do not have closedform probability density functions. Instead, they are usually 
represented by their characteristic function, given by

$$
\begin{aligned}
& \mathbb{E}\left[e^{i \theta X}\right] \\
& = \begin{cases}\exp \left\{-\gamma^{\alpha}|\theta|^{\alpha}\left(1-i \beta(\operatorname{sign} \theta) \tan \frac{\pi \alpha}{2}\right)+i \delta \theta\right\}, & \alpha \neq 1 \\
\exp \left\{-\gamma|\theta|\left(1+i \beta \frac{2}{\pi}(\operatorname{sign} \theta) \log |\theta|\right)+i \delta \theta\right\}, & \alpha=1\end{cases}
\end{aligned}
$$

The random variable $I$ arising from dynamic interference is complex, which leads to the concept of isotropic $\alpha$-stable random variables. Let $N_{1}, N_{2}$ be two symmetric $\alpha$-stable random variables. An isotropic $\alpha$-stable random variable $N=$ $N_{1}+i N_{2}$ then satisfies the following two conditions

C1: The random vector $\mathbf{N}=\left(N_{1}, N_{2}\right)^{T}$ is symmetric in $\mathbb{R}^{2}$; i.e., $\operatorname{Pr}(-\mathbf{N} \in A)=\operatorname{Pr}(\mathbf{N} \in A)$ for all Borel set $A \in \mathbb{R}^{2}$

C2: $e^{i \phi} N \stackrel{(d)}{=} N$ for any $\phi \in[0,2 \pi)$.

The random vector $\mathbf{N}$ is said to be induced by the isotropic $\alpha$-stable random variable $N$.

A key characterization of isotropic $\alpha$-stable random variables is in terms of a scale mixture representation.

Theorem 1. Let $0<\alpha<2$. A complex random variable $Z=Z_{1}+i Z_{2}$ is isotropic if and only if there are two independent and identically distributed zero-mean Gaussian random variables $G_{1}, G_{2}$ with variance $\sigma_{\mathrm{N}}^{2}$ and a random variable $A \sim S_{\frac{\alpha}{2}}\left((\cos (\pi \alpha / 4))^{2 / \alpha}, 1,0\right)$ independent of $\left(G_{1}, G_{2}\right)^{T}$ such that $\left(Z_{1}, Z_{2}\right)^{T}=A^{\frac{1}{2}}\left(G_{1}, G_{2}\right)^{T}$.

We also require the characteristic function of random vectors induced by isotropic $\alpha$-stable random variables. Since $\alpha$-stable random variables do not in general have closedform probability density functions, the characteristic function therefore plays a key role.

Property 1. The characteristic function of a random vector $\mathbf{Z}$ induced by an isotropic $\alpha$-stable random variable $Z(0<$ $\alpha<2$ ) is given by

$$
\phi_{\mathbf{Z}}(\boldsymbol{\theta})=\mathbb{E}\left[e^{i\left(\theta_{1} Z_{1}+\theta_{2} Z_{2}\right)}\right]=e^{-2^{-\alpha / 2} \sigma_{\mathbf{Z}}^{\alpha}|\boldsymbol{\theta}|^{\alpha}},
$$

where $\sigma_{\mathbf{Z}}$ corresponds to square root of the variance of the i.i.d Gaussian random variables in Theorem 1.

\section{B. Interference Characterization}

We now turn to characterizing the interference $I$, which is in fact an isotropic $\alpha$-stable random variable. To see this, let $z_{k}=h_{k} x_{k}$ and denote the real and imaginary parts as $z_{k, r}$ and $z_{k, i}$, respectively. The interference can then be written as

$$
I=\sum_{k=1}^{\infty} r_{k}^{-\eta / 2}\left(z_{k, r}+i z_{k, i}\right),
$$

where each device in $\Phi$ is indexed by an integer $k=1,2, \ldots$ and we can ignore the effect of the serving device by Slivnyak's theorem. Recall that the distances, $\left\{r_{k}\right\}_{k=1}^{\infty}$, are from points in a PPP to the origin. Using the mapping theorem, it follows that $r_{k}^{2}$ is a one-dimensional PPP with intensity $\lambda \pi$ [8, Theorem 1]. By an application of the LePage series representation of symmetric $\alpha$-stable random variables [14,
Theorem 1.4.2], it also follows that $I$ converges almost surely to

$$
I=Z_{r}+i Z_{i}
$$

where $Z_{r}$ and $Z_{i}$ are symmetric $4 / \eta$-stable random variables. By [14, Theorem 2.1.5(b)], the induced random vector $\mathbf{I}=$ $\left(Z_{r}, Z_{i}\right)^{T}$ is a symmetric $4 / \eta$-random vector, which implies that condition $\mathbf{C 1}$ holds.

To show that condition $\mathbf{C 2}$ holds, recall that $e^{i \phi} h_{k} x_{k} \stackrel{(d)}{=}$ $h_{k} x_{k}$ for any $\phi \in[0,2 \pi)$. This implies that $I$ is isotropic and hence $I$ is an isotropic $4 / \eta$-stable random variable.

In order to characterize the statistics of the interference $I$, all that remains is to obtain the parameter $\sigma_{\mathbf{N}}$ in the scale mixture representation stated in Theorem 1. Again using the LePage series representation in [14, Theorem 1.4.2], the scale parameters of the real and imaginary parts of $I$ are equal to $\left(\pi \lambda C_{\frac{\eta}{4}}^{-1} \mathbb{E}\left[\left|\operatorname{Re}\left(h_{k} x_{k}\right)\right|^{\frac{4}{\eta}}\right]\right)^{\frac{\eta}{4}}$. Using Property 1 , we then have

$$
\sigma_{\mathbf{N}}=\left(\pi \lambda C_{\frac{\eta}{4}}^{-1} \mathbb{E}\left[\left|\operatorname{Re}\left(h_{k} x_{k}\right)\right|^{\frac{4}{\eta}}\right]\right)^{\frac{\eta}{4}},
$$

where $C_{\frac{\eta}{4}}$ is given in (10).

In summary, the interference $I$ is characterized as follows.

Proposition 1. The interference $I$ is an isotropic $\alpha$-stable random variable, with $\alpha=\frac{4}{\eta}$ and parameter

$$
\sigma_{\mathbf{N}}=\left(\pi \lambda C_{\frac{4}{\eta}}^{-1} \mathbb{E}\left[\left|\operatorname{Re}\left(h_{k} x_{k}\right)\right|^{\frac{4}{\eta}}\right]\right)^{\frac{\eta}{4}},
$$

where $\Gamma(\cdot)$ is the Gamma function and

$$
C_{\alpha}= \begin{cases}\frac{1-\alpha}{\Gamma(2-\alpha) \cos (\pi \alpha / 2)}, & \text { if } \alpha \neq 1 \\ 2 / \pi, & \text { if } \alpha=1 .\end{cases}
$$

The main consequence of Proposition 1 is that the channel in (3) is a memoryless additive isotropic $\alpha$-stable noise $(A I \alpha S N)$ channel. Unlike circularly symmetric complex Gaussian noise, the real and imaginary parts of $I$ are not independenta consequence of Theorem 1. Therefore, it is not possible to treat an $A I \alpha S N$ channel as parallel real $\alpha$-stable noise channels. Instead, it is useful to view the $A I \alpha S N$ channel as a vector channel, where the real vector-valued noise is the vector induced by the isotropic $\alpha$-stable interference $I$.

\section{ACHiEvable RATES With Dynamic InTERFERENCE}

In this section, we derive the achievable rate for the access point at the origin. Unlike the power constrained Gaussian noise channel, tractable expressions are not known for the power constrained $A I \alpha S N$ channel. For this reason, it is desirable to consider alternative constraints.

One choice of constraints is the combination of amplitude and fractional moment constraints. In particular, the input signal $x_{d}$ in (3) is required to satisfy

$$
\begin{aligned}
\mathbb{E}\left[\left|\operatorname{Re}\left(x_{d}\right)\right|^{r}\right] & \leq c \\
\mathbb{E}\left[\left|\operatorname{Im}\left(x_{d}\right)\right|^{r}\right] & \leq c \\
\left|\operatorname{Re}\left(x_{d}\right)\right| & \leq A \\
\left|\operatorname{Im}\left(x_{d}\right)\right| & \leq A,
\end{aligned}
$$


where $0<r<\alpha$. Note that the presence of the amplitude constraint ensures that the input has finite moments, including power.

To characterize the capacity of the $A I \alpha S N$ channel in (3) subject to the constraints in (11), we proceed in two steps. First, we relax the amplitude constraints and consider the capacity optimization problem given by

$$
\begin{array}{cr}
\underset{\mu \in \mathcal{P}}{\operatorname{maximize}} & I(X ; y) \\
\text { subject to } & \mathbb{E}\left[|\operatorname{Re}(X)|^{r}\right] \leq c, \\
& \mathbb{E}\left[|\operatorname{Im}(X)|^{r}\right] \leq c,
\end{array}
$$

where $\mathcal{P}$ is the set of probability measures on $\mathbb{C}$ and $0<r<$ $\alpha$. The unique (see [9]) solution to (12) is lower bounded in the following theorem.

Theorem 2. For fixed $r_{d}$ and $h_{d}$, the capacity of the additive isotropic $\frac{4}{\eta}$-stable noise channel in (3) subject to the fractional moment constraints in (12) is lower bounded by:

$$
C_{L}=\frac{\eta}{4} \log \left(1+\frac{\left(\sqrt{2\left|r_{d}^{-\frac{\eta}{2}} h_{d}\right|^{2}}\left(\frac{c}{C\left(r, \frac{4}{\eta}\right)}\right)^{\frac{1}{r}}\right)^{\frac{4}{\eta}}}{\sigma_{\mathbf{N}}^{\frac{4}{\eta}}}\right),
$$

where $\Gamma(\cdot)$ is the Gamma function and

$$
C\left(r, \frac{4}{\eta}\right)=\frac{2^{r+1} \Gamma\left(\frac{r+1}{2}\right) \Gamma(-\eta r / 4)}{\frac{4}{\eta} \sqrt{\pi} \Gamma(-r / 2)} .
$$

Proof. We consider the case that $x_{d}$ is an isotropic $\alpha$-stable random variable satisfying the constraints in (12). By Theorem 5 in [9], the mutual information of the channel $Y=x_{d}+I$ is given by

$$
I\left(x_{d} ; Y\right)=\frac{\eta}{4} \log \left(1+\frac{\left(\sqrt{2}\left(\frac{c}{C\left(r, \frac{4}{\eta}\right)}\right)^{1 / r}\right)^{\frac{4}{\eta}}}{\sigma_{\mathbf{N}}^{\frac{4}{\eta}}}\right)
$$

The result then follows by observing that $r_{d}^{-\frac{\eta}{2}} h_{d} x_{d}$ is also an isotropic $\frac{4}{\eta}$-stable random variable with parameter $\left|r_{d}^{-\frac{\eta}{2}} h_{d}\right| \sigma_{\mathbf{N}}$ using the fact that $x_{d}$ is isotropic and [14, Property 1.2.3].

The achievable rate in Theorem 2 is obtained by using input signals that are isotropic $\alpha$-stable random variables, which does not satisfy the amplitude constraints in (11). The second step in characterizing the capacity of the $A I \alpha S N$ channel subject to (11) is therefore to consider a truncated isotropic $\alpha$-stable input. This guarantees the amplitude constraints are satisfied and, as we will show, yields a mutual information in the $A I \alpha S N$ channel that is well approximated by Theorem 2 for a sufficiently large truncation level $T$.

The truncated isotropic $\alpha$-stable random variables are defined as follows. Let $X$ be an isotropic $\alpha$-stable random variable, with real part $X_{r}$ and imaginary part $X_{i}$. The truncation of $X$, denoted by $X_{T}$, is given by

$$
X_{T}= \begin{cases}X, & \left|X_{r}\right| \leq T,\left|X_{i}\right| \leq T \\ \operatorname{sign}\left(X_{r}\right) T+i X_{i}, & \left|X_{r}\right|>T,\left|X_{i}\right| \leq T \\ X_{r}+i \operatorname{sign}\left(X_{i}\right) T, & \left|X_{r}\right| \leq T,\left|X_{i}\right|>T \\ \operatorname{sign}\left(X_{r}\right) T+i \operatorname{sign}\left(X_{i}\right) T, & \left|X_{r}\right|>T,\left|X_{i}\right|>T .\end{cases}
$$

Using the truncated isotropic $\alpha$-stable input, an achievable rate of the amplitude and fractional moment constrained $A I \alpha S N$ channel is obtained by evaluating the mutual information $I\left(y ; X_{T}\right)$, where $y$ is the output of the channel in (3). In fact, using a similar argument to that for the power constrained Gaussian noise channel [15], it is straightforward to show that all rates $R<I\left(y ; X_{T}\right)$ are achievable by using a codebook consisting of $2^{n R}$ codewords $W^{n}(1), \ldots, W^{n}\left(2^{n R}\right)$ with $W_{i}(w), i=1,2, \ldots, n, w=1,2, \ldots, 2^{n R}$ independent truncated isotropic $\alpha$-stable random variables.

Unfortunately, truncated isotropic $\alpha$-stable inputs do not lead to a closed-form mutual information for the channel in (3). In fact, only scaling laws for the capacity have been recently derived for real-valued inputs [16]. In order to characterize the achievable rates in the presence of dynamic interference, we therefore approximate $I\left(X_{T} ; y\right)$ by the lower bound in Theorem 2.

To verify that this approximation is indeed accurate, we numerically compute the mutual information $I\left(X_{T} ; y\right)$ and compare it with the result in Theorem 2 in Fig. 2 and Fig. 3 for $\alpha=1.7$ and $\alpha=1.3$, respectively. Observe that for a sufficiently large truncation level, the approximation based on Theorem 2 is in good agreement with $I\left(X_{T} ; y\right)$. Moreover, the achievable rate is significantly larger than the case of a Gaussian input. This suggests that Gaussian signaling is not necessarily desirable in the presence of dynamic interference; however, it is necessary to tune the truncation level numerically at present.

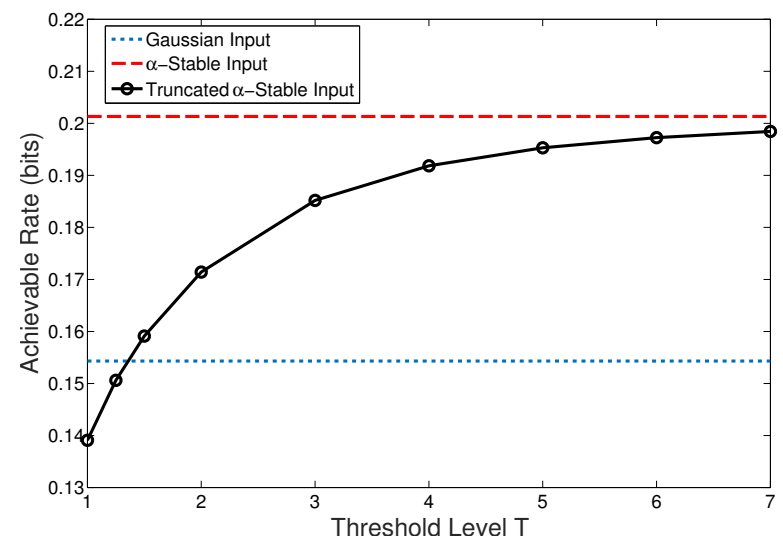

Fig. 2. Achievable rates for an $A I \alpha S N$ channel with $\alpha=1.7, \sigma_{\mathbf{N}}=0.1$ and a constraint $\mathbb{E}[|X|] \leq 1$. The curves correspond to a Gaussian input, an isotropic $\alpha$-stable input and a truncated isotropic $\alpha$-stable input (defined in (16)).

In light of the validity of the achievable rate approximation based on Theorem 2, we now turn to characterizing the 


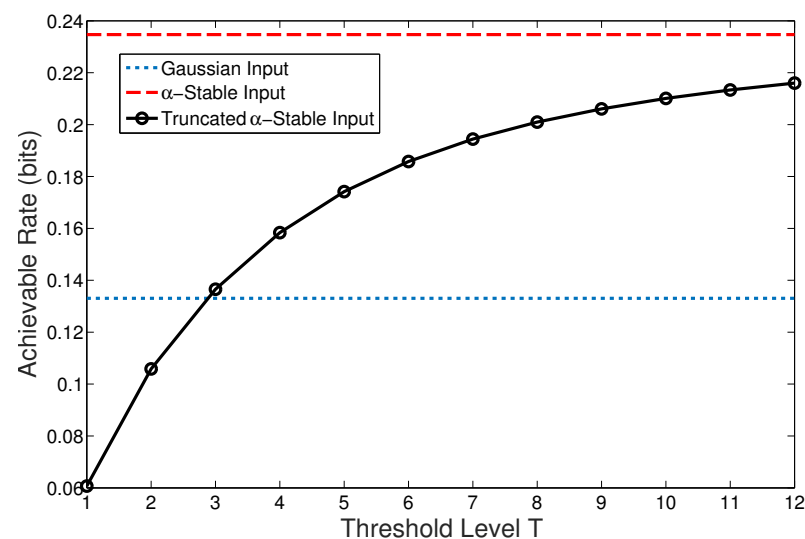

Fig. 3. Achievable rates for an $A I \alpha S N$ channel with $\alpha=1.3, \sigma_{\mathbf{N}}=0.5$ and a constraint $\mathbb{E}[|X|] \leq 1$. The curves correspond to a Gaussian input, an isotropic $\alpha$-stable input and a truncated isotropic $\alpha$-stable input (defined in (16)).

effect of device density in large-scale networks with dynamic interference.

\section{Area Spectral EFFiciency Analysis}

In this section, we investigate the effect of device density $\lambda$ on network performance. In particular, we study the area spectral efficiency, which is defined as the expected total rate per square meter. The area spectral efficiency captures the tradeoff between the distance between each device and its base station as well as the increasing interference as the device density increases. Formally, let $A_{1} \subset A_{2} \subset \cdots$ be a sequence of discs such that $\operatorname{Area}\left(A_{n}\right) \rightarrow \infty$ as $n \rightarrow \infty$. The area spectral efficiency is then given by

$$
\zeta=\lim _{n \rightarrow \infty} \frac{1}{\operatorname{Area}\left(A_{n}\right)} \mathbb{E}\left[\sum_{i \in \Phi\left(A_{n}\right)} R_{i}\left(A_{n}\right)\right],
$$

where $\Phi\left(A_{n}\right)$ is the PPP $\Phi$ restricted to the disc $A_{n}$ and $R_{i}\left(A_{n}\right)$ corresponds to the achievable rate with a truncated isotropic $\alpha$-stable input and devices in $\Phi\left(A_{n}\right)$.

The area spectral efficiency in the large-scale network detailed in Section II is given in the following theorem.

Theorem 3. The area spectral efficiency with device locations governed by a PPP, dynamic interference and truncated isotropic $\alpha$-stable inputs is given by

$$
\zeta=\lambda \mathbb{E}_{r_{d}, h_{d}}\left[R_{i}\right]
$$

where $R_{i}$ is the achievable rate with a truncated isotropic $\alpha$-stable input and devices in $\Phi$.

Proof. See Appendix A.

As observed in Section III, $R_{i}=I\left(y_{i} ; X_{T}\right)$ does not have a closed-form expression which makes characterizing the area spectral efficiency $\zeta$ challenging. To proceed, we exploit the approximation of $I\left(y_{i} ; X_{T}\right)$ based on Theorem 2. In particular, we obtain the following approximation for the area spectral efficiency

$$
\begin{aligned}
\zeta & \approx \lambda \mathbb{E}_{r_{d}, h_{d}}\left[\frac{\eta}{4} \log \left(1+\frac{\left(\sqrt{2\left|r_{d} h_{d}\right|^{2}}\left(\frac{c}{C\left(r, \frac{4}{\eta}\right)}\right)^{\frac{1}{r}}\right)^{\frac{4}{\eta}}}{\sigma_{\mathbf{N}}^{\frac{4}{\eta}}}\right)\right] \\
& =\frac{\lambda \eta}{4} \mathbb{E}_{r_{d}, h_{d}}\left[\log \left(1+\frac{\left(\sqrt{2\left|r_{d} h_{d}\right|^{2}}\left(\frac{c}{C\left(r, \frac{4}{\eta}\right)}\right)^{\frac{1}{r}}\right)^{\frac{4}{\eta}}}{\pi \lambda C_{\frac{\eta}{4}}^{-1} \mathbb{E}\left[\mid \operatorname{Re}\left(h_{k} x_{k}\right)^{\frac{4}{\eta}}\right]}\right)\right]
\end{aligned}
$$

which is tight when the truncation level for the input $T$ is sufficiently large. Further insight into the approximation error can be obtained numerically, such as in Fig. 2 and Fig. 3.

The expression in (19) provides insight into the effect of the device density $\lambda$. In particular, consider a function of the form

$$
f(\lambda)=\lambda \log \left(1+\frac{1}{\lambda}\right),
$$

which captures the dependence of the spatial rate density approximation in (19) on the device density $\lambda$. Since we are interested in studying the impact of device density, an application of Leibniz's rule in (19) shows that it is sufficient to consider (20). Evaluating the derivative yields $f^{\prime}(\lambda)=$ $\log \left(1+\frac{1}{\lambda}\right)-\frac{1}{1+\lambda}$.

Since $\log x>1-\frac{1}{x}$ for $x>1$, it follows that $\log \left(1+\frac{1}{\lambda}\right)>$ $\frac{1}{1+\lambda}$ and hence for $\lambda>0, f^{\prime}(\lambda)>0$. This implies that the area spectral efficiency $\zeta$ is an increasing function of the density $\lambda$ (illustrated in Fig. 4). We therefore conclude that dense networks maximize the area spectral efficiency. We remark that dense networks are also desirable for slowly varying active interferer sets [11]. This implies that although the optimal signaling strategy for each link is no longer Gaussian, the basic network structure is the same both for dynamic interference and interference arising from a slowly varying active interferer set.

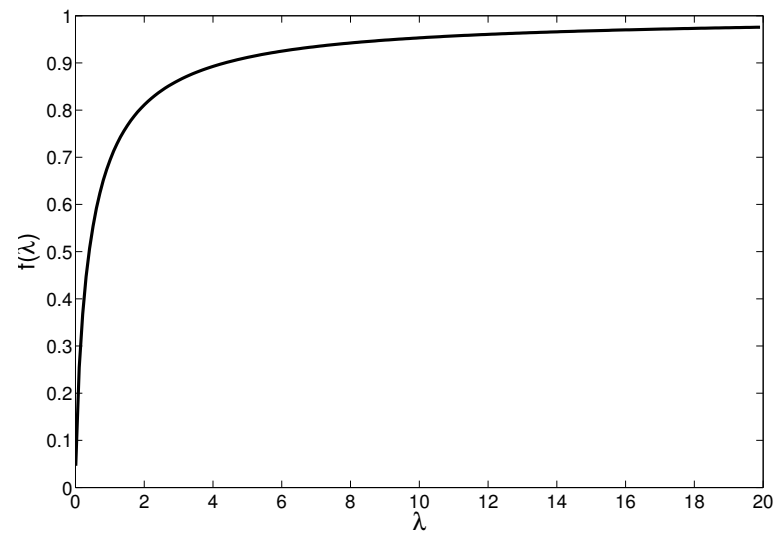

Fig. 4. Plot of $f(\lambda)$ in (20). 


\section{Conclusions}

Rapid changes in the active transmitter set is a characteristic of wireless communication networks with very short transmissions, which arises in M2M communications. A consequence of the rapid changes in the active transmitter set is that the interference is dynamic. We have shown that dynamic interference is not Gaussian. In fact, the interference is isotropic $\alpha$-stable for large scale networks with interferers located according to a PPP.

A key question is therefore what rates are achievable in the presence of isotropic $\alpha$-stable interference. In this paper, we have derived a closed-form expression for the achievable rate with an isotropic $\alpha$-stable input. Moreover, numerical results suggest that it well approximates the achievable rate when the input signal is truncated, for sufficiently large truncation levels.

In order to establish the effect of device density in large scale networks with dynamic interference, the area spectral efficiency was studied. We showed that dense networks optimize the area spectral efficiency, consistent with analysis for networks with slowly varying active transmitter sets.

\section{APPENDIX A \\ PROOF OF THEOREM 3}

In order to compute the area spectral efficiency $\zeta$, observe that the random variables $R_{i}\left(A_{n}\right)$ are identically distributed (but not independent) since the distances $r_{d}$ are independent and identically distributed, and the locations of the devices are independently and uniformly distributed in $A_{n}$ conditioned on the number of devices $N\left(A_{n}\right)$ in $A_{n}$ [17]. By the strong law of large numbers for PPPs [18], $\frac{N\left(A_{n}\right)}{\operatorname{Area}\left(A_{n}\right)} \cong \lambda$ a.s. as $n \rightarrow \infty$. Let $\epsilon>0$, it then follows that

$$
\begin{aligned}
\zeta= & \lim _{n \rightarrow \infty} \frac{1}{\operatorname{Area}\left(A_{n}\right)} \mathbb{E}\left[\sum_{i=1}^{\operatorname{Area}\left(A_{n}\right) \frac{N\left(A_{n}\right)}{\operatorname{Area}\left(A_{n}\right)}} R_{i}\left(A_{n}\right)\right] \\
= & \lim _{n \rightarrow \infty} \frac{1}{\operatorname{Area}\left(A_{n}\right)} \\
& \times\left(\mathbb{E}\left[\sum_{i=1}^{\left[\left\lfloor\operatorname{Area}\left(A_{n}\right) \lambda_{1}\right\rfloor\right.} R_{i}\left(A_{n}\right) \mid \lambda_{1} \in[\lambda-\epsilon, \lambda+\epsilon]\right]\right. \\
& \times \operatorname{Pr}\left(\lambda_{1} \in[\lambda-\epsilon, \lambda+\epsilon]\right) \\
& +\mathbb{E}\left[\sum_{i=1}^{\left\lfloor\operatorname{Area}\left(A_{n}\right) \lambda_{1}\right\rfloor} R_{i}\left(A_{n}\right) \mid \lambda_{1} \notin[\lambda-\epsilon, \lambda+\epsilon]\right] \\
& \left.\times \operatorname{Pr}\left(\lambda_{1} \notin[\lambda-\epsilon, \lambda+\epsilon]\right)\right)
\end{aligned}
$$

A direct consequence of the strong law of large numbers of PPPs is that as $n \rightarrow \infty, \operatorname{Pr}\left(\lambda_{1} \in[\lambda-\epsilon, \lambda+\epsilon]\right) \rightarrow 1$.

Next, for fixed large $n$ select $A_{n}$ such that $\lambda A_{n}$ is an integer and $\epsilon>0$ sufficiently small such that $\lambda A_{n}$ is the only integer in $[\lambda-\epsilon, \lambda+\epsilon]$. It then follows that

$$
\begin{aligned}
\zeta & =\lim _{n \rightarrow \infty} \frac{1}{\operatorname{Area}\left(A_{n}\right)} \operatorname{Area}\left(A_{n}\right) \lambda \mathbb{E}\left[R_{i}\left(A_{n}\right)\right] \\
& =\lambda \lim _{n \rightarrow \infty} \mathbb{E}\left[R_{i}\left(A_{n}\right)\right] .
\end{aligned}
$$

To evaluate $\lim _{n \rightarrow \infty} \mathbb{E}\left[R_{i}\left(A_{n}\right)\right]$, let $y_{i, A_{n}}$ be the received signal at the access point served by the $i$-th device in $\Phi\left(A_{n}\right)$. For fixed $r_{d}, h_{d}, R_{i}\left(A_{n}\right)=I\left(y_{i, A_{n}} ; X_{T}\right)$. From the LePage series representation of the interference in (6), it follows that the signal received by the access point served by the $i$-th device in $\Phi$ satisfies $y_{i} \stackrel{(d)}{=} r_{d}^{-\frac{\eta}{2}} h_{d} X_{T}+I$, a.s. as $n \rightarrow \infty$.

Since the conditions in [19, Theorem 1] hold, it follows that for fixed $r_{d}, h_{d}$ we have $I\left(y_{i, A_{n}} ; X_{T}\right) \rightarrow I\left(y_{i} ; X_{T}\right)$ as $n \rightarrow \infty$. As $R_{i}\left(A_{n}\right)$ is positive and $R_{i}\left(A_{n}\right) \rightarrow R_{i}$ as $n \rightarrow \infty$, we then obtain the desired result.

\section{REFERENCES}

[1] A. Al-Fuqaha, M. Guizani, M. Mohammadi, M. Aledhari, and M. Ayyash, "Internet of things: A survey on enabling technologies, protocols, and applications," IEEE Communications Surveys \& Tutorials, vol. 17, no. 4, pp. 2347-2376, 2015.

[2] M. Tolstrup, Indoor Radio Planning: A Practical Guide for 2G, $3 G$ and 4G. John Wiley \& Sons, Inc., 2015.

[3] Digi, "Efficient data transfer over cellular networks." White Paper, 2009.

[4] P. Pinto and M. Win, "Communication in a Poisson field of interfererspart I: interference distribution and error probability," IEEE Transactions on Wireless Communications, vol. 9, no. 7, pp. 2176-2186, 2010.

[5] P. Pinto and M. Win, "Communication in a Poisson field of interfererspart II: channel capacity and interference spectrum," IEEE Transactions on Wireless Communications, vol. 9, no. 7, pp. 2187-2195, 2010.

[6] K. Gulati, B. Evans, J. Andrews, and K. Tinsley, "Statistics of co-channel interference in a field of Poisson-Poisson clustered interferers," IEEE Transactions on Signal Processing, vol. 58, no. 12, pp. 6207-6222, 2010.

[7] X. Yang and A. Petropulu, "Co-channel interference modeling and analysis in a Poisson field of interferers in wireless communications," IEEE Transactions on Signal Processing, vol. 51, no. 1, pp. 64-76, 2003.

[8] J. Ilow and D. Hatzinakos, "Analytic alpha-stable noise modeling in a poisson field of interfers or scatterers," IEEE Transactions on Signal Processing, vol. 46, no. 6, pp. 1601-1611, 1998.

[9] M. Egan, M. de Freitas, L. Clavier, A. Goupil, G. Peters, and N. Azzaoui, "Achievable rates for additive isotropic alpha-stable noise channels," in Proc. of the IEEE International Symposium on Information Theory, 2016.

[10] M.-S. Alouini and A. Goldsmith, "Area spectral efficiency of cellular mobile radio systems," IEEE Transactions on Vehicular Technology, vol. 48, no. 4, pp. 1047-1066, 1999.

[11] M. Ding, P. Wang, D. López-Pérez, G. Mao, and Z. Lin, "Performance impact of LoS and NLos transmissions in dense cellular networks," IEEE Transactions on Wireless Communications, vol. 15, no. 3, pp. 23652380, 2016

[12] R. Igual Pérez, Platform Hardware/Software for the energy optimization in a node of wireless sensor networks. PhD thesis, Université Lille 1 Sciences et Technologies, Lille, June 2016.

[13] M. de Freitas, M. Egan, L. Clavier, G. Peters, and N. Azzaoui, "Capacity bounds for additive symmetric alpha-stable noise channels," To appear in IEEE Transactions on Information Theory.

[14] G. Samorodnitsky and M. Taqqu, Stable Non-Gaussian Random Processes. Chapman and Hall, 1994.

[15] T. Cover and J. Thomas, Elements of Information Theory, Second Edition. John Wiley \& Sons, Inc., 2006.

[16] M. Egan, S. Perlaza, and V. Kungurtsev, "Capacity sensitivity in additive non-gaussian noise channels," in Proc. IEEE International Symposium on Information Theory, 2017.

[17] D. Daley and D. Vere-Jones, An Introduction to the Theory of Point Processes. Springer, 2003.

[18] M. Haenggi, Stochastic Geometry for Wireless Networks. Cambridge University Press, 2013.

[19] J. Fahs and I. Abou-Faycal, "On the finiteness of the capacity of continuous channels," IEEE Transactions on Communications, vol. 64, no. 1, pp. 166-173, 2016. 